\title{
Feature Ranking of Active Region Source Properties in Solar Flare Forecasting and the Uncompromised Stochasticity of Flare Occurrence
}

\author{
${\text { Cristina } \text { Campi }^{1} \text {, Federico Benvenuto }}^{2}$ (1) , Anna Maria Massone ${ }^{2,3}$, D. Shaun Bloomfield ${ }^{4}$ (10), Manolis K. Georgoulis ${ }^{5,6}$ (1), and \\ Michele Piana ${ }^{2,3}$ \\ ${ }^{1}$ Dipartimento di Matematica “Tullio Levi Civita," Università di Padova, via Trieste 63 I-35121 Padova, Italy; cristina.campi@unipd.it \\ 2 Dipartimento di Matematica, Università di Genova, via Dodecaneso 35 I-16146 Genova, Italy; benvenuto@dima.unige.it, massone@dima.unige.it, \\ piana@dima.unige.it \\ ${ }^{3}$ CNR - SPIN Genova, via Dodecaneso 33 I-16146 Genova, Italy \\ ${ }^{4}$ Department of Mathematics, Physics and Electrical Engineering, Northumbria University, Newcastle upon Tyne, NE1 8ST, UK; \\ shaun.bloomfield@northumbria.ac.uk \\ ${ }^{5}$ Department of Physics and Astronomy, Georgia State University, Atlanta (GA), USA; manolis.georgoulis@academyofathens.gr \\ ${ }^{6}$ RCAAM of the Academy of Athens, Athens, Greece \\ Received 2019 June 27; revised 2019 August 12; accepted 2019 August 14; published 2019 September 30
}

\begin{abstract}
Solar flares originate from magnetically active regions (ARs) but not all solar ARs give rise to a flare. Therefore, the challenge of solar flare prediction benefits from an intelligent computational analysis of physics-based properties extracted from AR observables, most commonly line-of-sight or vector magnetograms of the active region photosphere. For the purpose of flare forecasting, this study utilizes an unprecedented 171 flare-predictive AR properties, mainly inferred by the Helioseismic and Magnetic Imager on board the Solar Dynamics Observatory (SDO/HMI) in the course of the European Union Horizon 2020 FLARECAST project. Using two different supervised machine-learning methods that allow feature ranking as a function of predictive capability, we show that (i) an objective training and testing process is paramount for the performance of every supervised machine-learning method; (ii) most properties include overlapping information and are therefore highly redundant for flare prediction; (iii) solar flare prediction is still—and will likely remain—a predominantly probabilistic challenge.
\end{abstract}

Unified Astronomy Thesaurus concepts: Astronomy data analysis (1858); Neural networks (1933); Solar flares (1496); Solar activity (1475); Solar active region magnetic fields (1975)

\section{Introduction}

Solar flares are the most explosive events in the heliosphere, releasing in an abrupt way up to $10^{33} \mathrm{erg}$ of energy in a time interval typically ranging between 10 and $1000 \mathrm{~s}$ (Benz 2017). This energy is previously stored in specific magnetic configurations and, when magnetic reconnection occurs (Priest \& Forbes 2002), it is transformed into mass acceleration, heating, and electromagnetic radiation at all wavelengths. It is also established that flares are a major space weather agent in the heliosphere (Schwenn 2006), while, as secondary effects through their correlation with coronal mass ejections, they induce geospace and ionospheric disturbances, malfunctions, and impairments on technologies in the geosphere, such as flight navigation, satellite communication, and power grid distribution.

Solar flare forecasting is a prominent discipline (Gallagher et al. 2002; Georgoulis \& Rust 2007; Li et al. 2007, 2008; Schrijver 2007; Barnes \& Leka 2008; Wang et al. 2008; Colak \& Qahwaji 2009; Yu et al. 2009; Ahmed et al. 2013; Bobra \& Couvidat 2015; Barnes et al. 2016; McCloskey et al. 2017; Murray et al. 2017; Sadykov \& Kosovichev 2017; Benvenuto et al. 2018; Huang et al. 2018; Massone et al. 2018; Nishizuka et al. 2018; Park et al. 2018) within the recent field of space weather forecasting that relies on the availability of two ingredients; one observational and one computational. First, it is well-established that solar active regions (ARs) exclusively host major flares and therefore flare prediction needs experimental data on AR properties, associated to the photospheric and coronal magnetic field; however, coronal information has only recently started being used in the form of EUV images given as input to a deep learning network by Nishizuka et al. (2018). Second, this information on AR magnetic properties can be processed for prediction purposes by means of a computational method for data analysis; machine learning has recently offered strong candidates for such methods.

Since February 2010, the Helioseismic and Magnetic Imager on board the Solar Dynamics Observatory (SDO/HMI; Scherrer et al. 2012) is providing both line-of-sight and vector magnetograms of the full solar disk at a (vector magnetogram) cadence of 12 minutes. SDO/HMI magnetograms can be used for solar flare prediction according to two different approaches. First, HMI magnetograms are utilized to calculate a variety of properties either from the line-of-sight component only, from the radial component only, or from all three vector components. Various single-valued quantities, hereafter referred to as features, can be calculated from these property images through a variety of techniques (e.g., thresholding, feature recognition, etc.), such that calculation of one physical property may provide multiple features as inputs to machine learning (i.e., image maximum, total, and moments). Of course, additional features that are not derived from property images may also contribute to the input data set. Second, from a deep learning perspective, HMI images can be given as input to Convolutional Neural Networks that automatically perform a probabilistic forecasting. This present paper follows the first approach, and this is for several reasons. First, we had at our disposal the property extraction power provided by the algorithms developed within the Horizon 2020 FLARECAST project (http:// flarecast.eu), which generated data sets of almost 200 features determined from properties extracted from photospheric $S D O /$ 
HMI vector magnetograms. This database of features probably represents the highest data dimensionality currently available for flare forecasting purposes. Second, one of the objectives of our research was to determine to what extent $\mathrm{AR}$ properties are redundant when forecasting flares, and a straightforward way to do this is by ranking the extracted features according to their predictive capability. Finally, so far most publications in flare prediction utilize feature-based machine-learning methods, so another objective of this paper is to investigate how data preparation (and, specifically, the preparation of the training set in the case of supervised algorithms) impacts the prediction scores. Specifically, the analysis performed in this paper relies on two supervised machine-learning algorithms that combine prediction with feature ranking, namely hybrid LASSO (Benvenuto et al. 2018) and Random Forest (RF; Breiman 2001). However, two other methods of this kind, namely logit (Wu et al. 2009) and a support vector machine for classification (Cortes \& Vapnik 1995), have been also applied to the same data sets for verification purposes, with coherent results.

The content of the paper is as follows. Section 2 overviews the data analysis procedure, describing in detail the features used for prediction, the data preparation process, and key aspects of the machine-learning methods adopted. Section 3 contains the results of the analysis, while Section 4 discusses these results. Our conclusions are offered in Section 5.

\section{Methods}

\subsection{Data and Features}

Our analysis relies on the Near-Realtime Space Weather HMI Archive Patch (SHARP) data product of the HMI database (Bobra et al. 2014). These data comprise 2D images of continuum intensity, the full three-component magnetic field vector, and the line-of-sight component of each photospheric HARP. We then made use of property extraction algorithms developed by the FLARECAST Consortium in order to construct a property database made of property vectors comprising up to 171 components. FLARECAST algorithms first extracted the following 167 features, often duplicating the property calculation step on $B_{\text {los }}$ and $B_{\text {radial }}$ input data, as per the findings of Guerra et al. (2018):

1. Schrijver's $R$-value (Schrijver 2007): one property yielding a total of two features.

2. Multifractal structure and function spectrum on a $2 \mathrm{D}$ image: two properties yielding a total of four features.

3. Falconer's total free magnetic energy proxy $W L_{S G}$ (Falconer et al. 2008): one property yielding a total of two features.

4. Sum of the horizontal magnetic gradient, $G_{S}$, and the separation of opposite-polarity sunspot subgroups, $S_{l-f}$ (Korsos \& Erdelyi 2016): two properties yielding a total of four features.

5. Spectral power indices extracted by means of the Fourier transform and of a continuous wavelet transform: one property yielding a total of four features.

6. Magnetic polarity inversion line (MPIL) characteristics: three properties yielding a total of six features.

7. Effective connected magnetic field strength $\left(B_{\text {eff }}\right)$ : one property yielding a total of two features.

8. Vertical decay index of potential field: four properties yielding a total of eight features.
9. Nonneutralized electric currents: one property yielding one feature.

10. Ising energy $(E)$ : one property yielding a total of four features.

11. Fractal dimension $(D)$ : one property yielding a total of two features.

12. Flow field characteristics: six properties yielding a total of 16 features.

13. Magnetic helicity and energy injection rate: 14 properties yielding a total of 14 features.

14. SHARP keywords calculated from their corresponding vector and line-of-sight magnetograms: 16 properties yielding a total of 100 features (including the maximum, total, median, mean, standard deviation, skewness and kurtosis over the SHARP field-of-view).

SHARP ARs are associated to solar flares of GOES class C1 and above $(\mathrm{C} 1+)$ and solar flares of GOES class M1 and above $(\mathrm{M} 1+)$ by means of a standard procedure. It is first verified whether the SHARP data contain NOAA-numbered regions (i.e., sunspot groups) by comparison with NOAA's daily Solar Region Summary (SRS) file immediately before the SHARP observations. Then, if any NOAA number is assigned to the SHARP data, the process searches the NOAA/SWPC daily events lists for GOES flares occurring in the same source region during the entire disk passage. Once the flare association is realized, the following four details become available for all flares and these are used in assigning flare outcome labels:

1. GOES peak magnitude $\left(F_{M}\right)$.

2. Time difference (in seconds) between the SHARP observation time and the flare start time $\left(\tau_{s}\right)$.

3. Time difference (in seconds) between the SHARP observation time and the flare peak time $\left(\tau_{p}\right)$.

4. Time difference (in seconds) between the SHARP observation time and the flare end time $\left(\tau_{e}\right)$.

Eventually, this analysis provides 167 features extracted from the HMI images. Four further features come from the NOAA/ SRS database: the mean heliographic longitude and latitude of each AR, a binary label encoding the presence of a flare in the past $24 \mathrm{hr}$ and the flare index of events occurring within the past $24 \mathrm{hr}$. A summary of all resulting features considered in the analysis can be found at both the url https://api.flarecast. eu/property/ui/ and in Tables 3-5 in the Appendix.

\subsection{Data Preparation}

The experiment designed in this paper relies on supervised machine learning, which requires appropriate historical sets to train the prediction networks. To enforce consistency in time and robustness of our tests, we constructed four training sets, each one corresponding to a specific forecast issuing time expressed as universal time [UT], namely 00:00, 06:00, 12:00, and 18:00. For each issuing time we considered the set of $S D O / \mathrm{HMI}$ images recorded at that time in the range of days between 2012 September 14 and 2016 April 30, with $24 \mathrm{hr}$ sampling. While filling up the training set we took care to focus on ARs rather than on feature vectors. In fact, around twothirds of ARs were randomly extracted from the set of all ARs belonging to a specific issuing time and the 171-dimensional feature vectors associated to each AR were labeled by annotating whether a GOES $\mathrm{C} 1+$ flare occurred in the next $24 \mathrm{hr}$. The set of feature vectors associated to the remaining 
one-third of ARs was not labeled and was provided as a test set for experiments on supervised learning algorithms trained on the training set. In this manner, training and testing do not overlap in any way, either in time or in terms of ARs examined. We finally point out that, for each issuing time, the random, complete separation of ARs into training and test sets was replicated 100 times to enable statistical robustness of the results. A similar procedure was implemented to generate training sets to use for the prediction of GOES M1+ flares. The reason why we did not consider the prediction of flares with class above $\mathrm{X}$ is because they are extremely seldom in the database (less than $0.2 \%$ of the overall point-in-time events in the original training set). $\mathrm{C} 1+$ and $\mathrm{M} 1+$ flares are around $26 \%$ and $4 \%$ of the set content, respectively.

\subsection{Prediction and Feature Ranking Methods}

Two different machine-learning methods, namely hybrid LASSO (HLA) and RF are utilized in this paper, for both performing the binary prediction of the flare occurrence and for additionally identifying the effectiveness with which the different features contribute to the prediction.

LASSO methods (Tibshirani 1996) are intrinsically regression methods and therefore they are not originally conceived for applications that require a binary YES/NO output. However, in Benvenuto et al. (2018) a threshold optimization is introduced to the LASSO outcome in order to realize classification by means of fuzzy clustering (Bezdek et al. 1984). The idea of HLA is therefore to use LASSO in the first step in order to promote sparsity and to realize feature selection; this step provides an optimal estimate of the model parameters and corresponding predicted output. In the second step, Fuzzy C-Means is applied for clustering the predicted output in two classes. The main advantage of this approach is in the use of fuzzy clustering to automatically classify the regression output in two classes. Indeed, fuzzy clustering identifies flaring/nonflaring events with a thresholding procedure that is data-adaptive and completely operator-independent. Details about HLA as implemented in the present paper can be found in Benvenuto et al. (2018).

RF belongs to the family of the ensemble methods, i.e., methods that make use of a combination of different learning models to increase the classification accuracy. In particular, RF works as a large collection of decorrelated decision trees. In fact, here the training set is randomly divided into 10 subsets and for each subset a separate decision tree is built. Each decision tree is then used to classify an incoming unlabeled sample. If correctly implemented, RF can be used as feature rankers. In fact, the relative depth of a feature used as a decision node in a tree can be identified as the relative importance of that feature with respect to the predictability of the target variable. Features used at the top of the tree contribute to the final prediction decision of a larger fraction of the input samples. The expected fraction of the samples they contribute to can thus be used as an estimate of the relative importance of the features. Details about RF as implemented in the present paper can be found in Breiman (2001).

Once the two machine-learning methods have been applied to the input data, predictors are ranked by using recursive feature elimination (RFE). This iterative procedure can be summarized as follows:

1. Train the classifier.

2. Compute the ranking for all features.

3. Remove the feature with the smallest ranking.
Details about RFE as implemented in the present paper can be found in Guyon et al. (2002).

\section{Results}

The effectiveness of the prediction was assessed by skill scores computed on the previously unseen test sets. Following suggestions in Bloomfield et al. (2012), we chose to use the true skill statistic (TSS) and the Heidke skill score (HSS), assuming them as representative among skill scores existing in the literature. This said, although not shown here, we performed the analysis using the false alarm ratio, probability of detection, and accuracy metrics, obtaining similar results in terms of the relative forecasting effectiveness of the two machine-learning methods. We point out that all these scores are computed by means of binary predictions applied to the test set. However, as noted in Section 2.3, LASSO and RF are regression methods providing as outcome real positive numbers that can be interpreted in a probabilistic sense and, in our approach, the transformation of these variables into dichotomous yes/no responses is accomplished by applying a fuzzy clustering technique against the regression outcomes. Other works typically apply an arbitrary probability threshold, $P_{\text {th }}$, of 0.5 to create dichotomous forecasts (Leka et al. 2019a, 2019b), although it should be noted that discriminating thresholds optimized on TSS should find $P_{\text {th }}$ values close to the climatology (i.e., the average flare-day rate; Bloomfield et al. 2012; Barnes et al. 2016). Figure 1 shows a comparison between the thresholding performances of the clustering technique and the ones provided by the optimization of TSS and HSS and by the use of an ROC curve. Interestingly, the two hybrid regression/clustering approaches provide similar results, which are rather conservative and rather close to the ones achieved by optimizing the HSS, especially in the case of the prediction of $\mathrm{C} 1+$ flares. Furthermore, the ROC curve method relies on cutoff values computed by means of the Youden index (Youden 1950), which formally leads to the maximization of the TSS; in fact the figure shows that the two values are always very close and the small differences are just related to the different numerical way the thresholding-search schemes were implemented. For $\mathrm{C} 1+$ flares, our hybrid approach results in $P_{\text {th }} \approx 0.4$ for both HLA and RF, meaning that our $\mathrm{C} 1+\mathrm{TSS}$ values are (probably) more comparable to those whose probabilities are converted to dichotomous forecasts using $P_{\text {th }}=0.5$ (as our $P_{\text {th }}$ lies closer to 0.5 than the average $\mathrm{C} 1+$ flare-day rate of $\sim 0.26$ ). The situation is more complex for $\mathrm{M} 1+$ flares, however, as the average $P_{\text {th }}$ found by the fuzzy-clustered HLA method is almost equivalent to that optimized on TSS (i.e., approaching the average flare-day rate of 0.04) while for the fuzzy-clustered RF method it instead occupies greater values that lie between the TSS and HSS optimized cases.

The averages and standard deviations of the TSS and HSS values over 100 random realizations of the training/test sets for both prediction methods are shown in Table 1. In the case of HSS, the reliability of average values may be challenged by inconsistent flare/no-flare imbalance ratio across the 100 realizations. However, we have a posteriori checked the sample statistics of the 100 random realizations: average flare/no-flare imbalance ratios across the 100 test sets are $\sim 0.34$ for $\mathrm{C} 1+$ events and $\sim 0.04$ for $\mathrm{M} 1+$ events, with relative standard deviations that are $<16 \%$ and $<27 \%$ of these values, respectively (reflecting the 

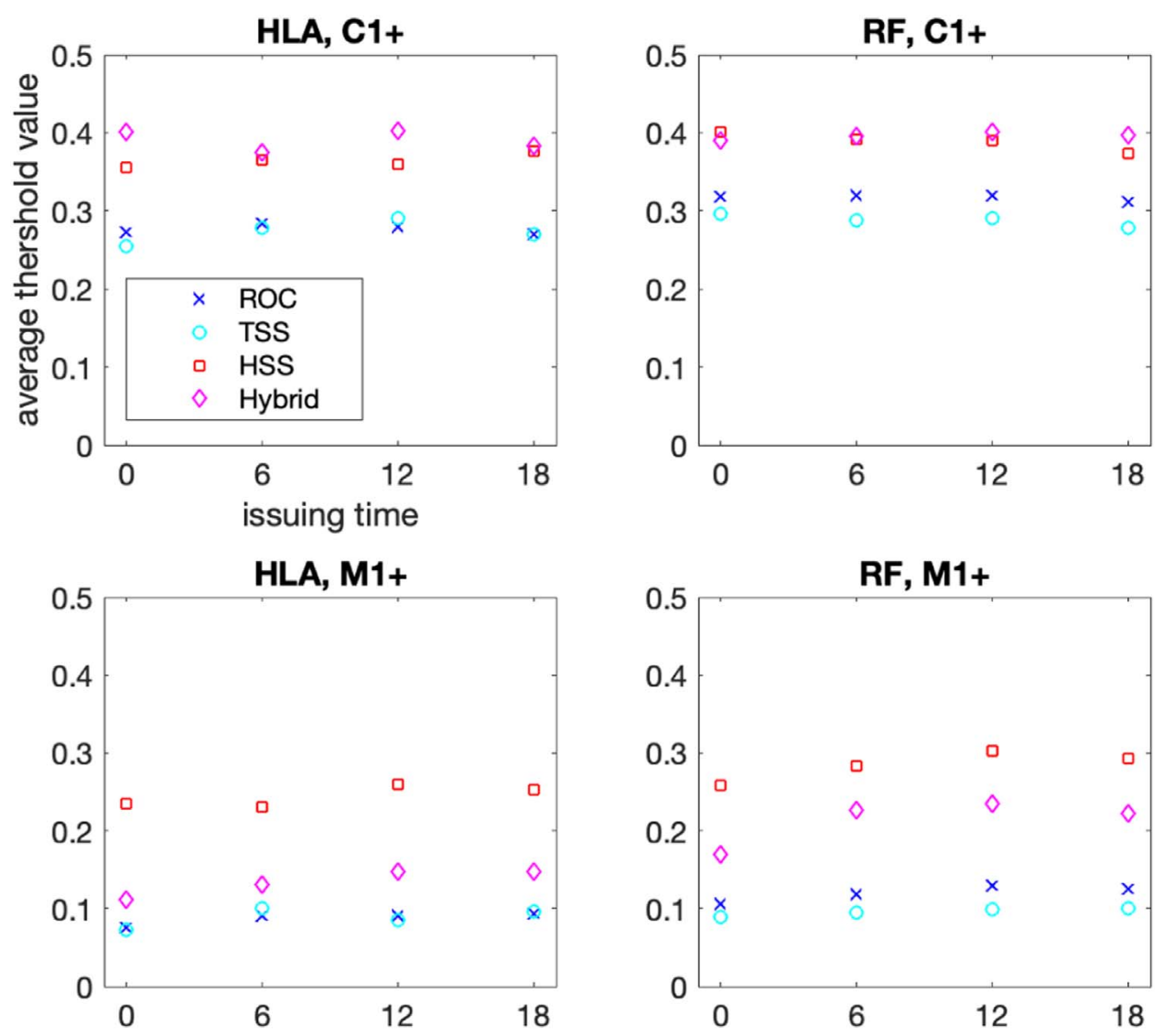

Figure 1. Probability threshold values, $P_{\text {th }}$, averaged over the 100 realizations of the training set. In each panel, symbols indicate the approach applied: hybrid fuzzy clustering (diamonds); HSS optimization (squares); TSS optimization (circles); ROC curve YoudenÕs index optimization (crosses). Top row: prediction of C1+ flares with LASSO and RF (left and right panels, respectively). Bottom row: prediction of M1+flares with LASSO and RF (left and right panels, respectively).

Table 1

Average TSS- and HSS-values, Along with Applicable Standard Deviations, Over the Outcomes of HLA and RF as Applied Against 100 Random Realizations of the Training/test Sets

\begin{tabular}{lcccc}
\hline \hline & Test Set-C1+ & Test Set-C1+ & Test Set-M1+ & TSS \\
\hline 00:00:00UT & TSS & HSS & HSS \\
\hline HLA & $0.48 \pm 0.06$ & $0.51 \pm 0.05$ & $0.56 \pm 0.14$ & $0.48 \pm 0.14$ \\
RF & $0.53 \pm 0.05$ & $0.52 \pm 0.04$ & TSS & $0.27 \pm 0.06$ \\
\hline 06:00:00UT & TSS & HSS & $0.67 \pm 0.05$ & HSS \\
\hline HLA & $0.53 \pm 0.03$ & $0.54 \pm 0.03$ & $0.49 \pm 0.08$ & $0.35 \pm 0.04$ \\
RF & $0.54 \pm 0.03$ & $0.54 \pm 0.03$ & TSS & $0.42 \pm 0.06$ \\
\hline 12:00:00UT & TSS & HSS & $0.66 \pm 0.06$ & HSS \\
\hline HLA & $0.51 \pm 0.04$ & $0.54 \pm 0.03$ & $0.51 \pm 0.09$ & TSS \\
RF & $0.53 \pm 0.03$ & $0.53 \pm 0.03$ & $0.38 \pm 0.04$ \\
\hline 18:00:00UT & TSS & HSS & $0.64 \pm 0.07$ & HSS \\
\hline HLA & $0.54 \pm 0.04$ & $0.55 \pm 0.03$ & $0.53 \pm 0.09$ \\
RF & $0.55 \pm 0.03$ & $0.55 \pm 0.03$ & $0.39 \pm 0.04$ \\
\hline
\end{tabular}

largest relative standard deviations for the four separate UT issuing times considered here).

Focusing then on the feature ranking process, the boxplots in Figures 2 and 3 show the top 10 features ordered by their mean RFE ranking, obtained by HLA and RF over the 100 random realizations for each of the four forecast issuing times. Specifically, Figure 2 refers to the prediction of GOES C1+ flares, while Figure 3 refers to the prediction of GOES M1+ flares.

From these results it becomes possible to assess the impact of feature selection on the prediction performance, by computing specific skill scores and statistics in a cumulative way. The panels in Figures 4 and 5 plot the TSS values obtained by HLA and RF in the case of one specific data set 

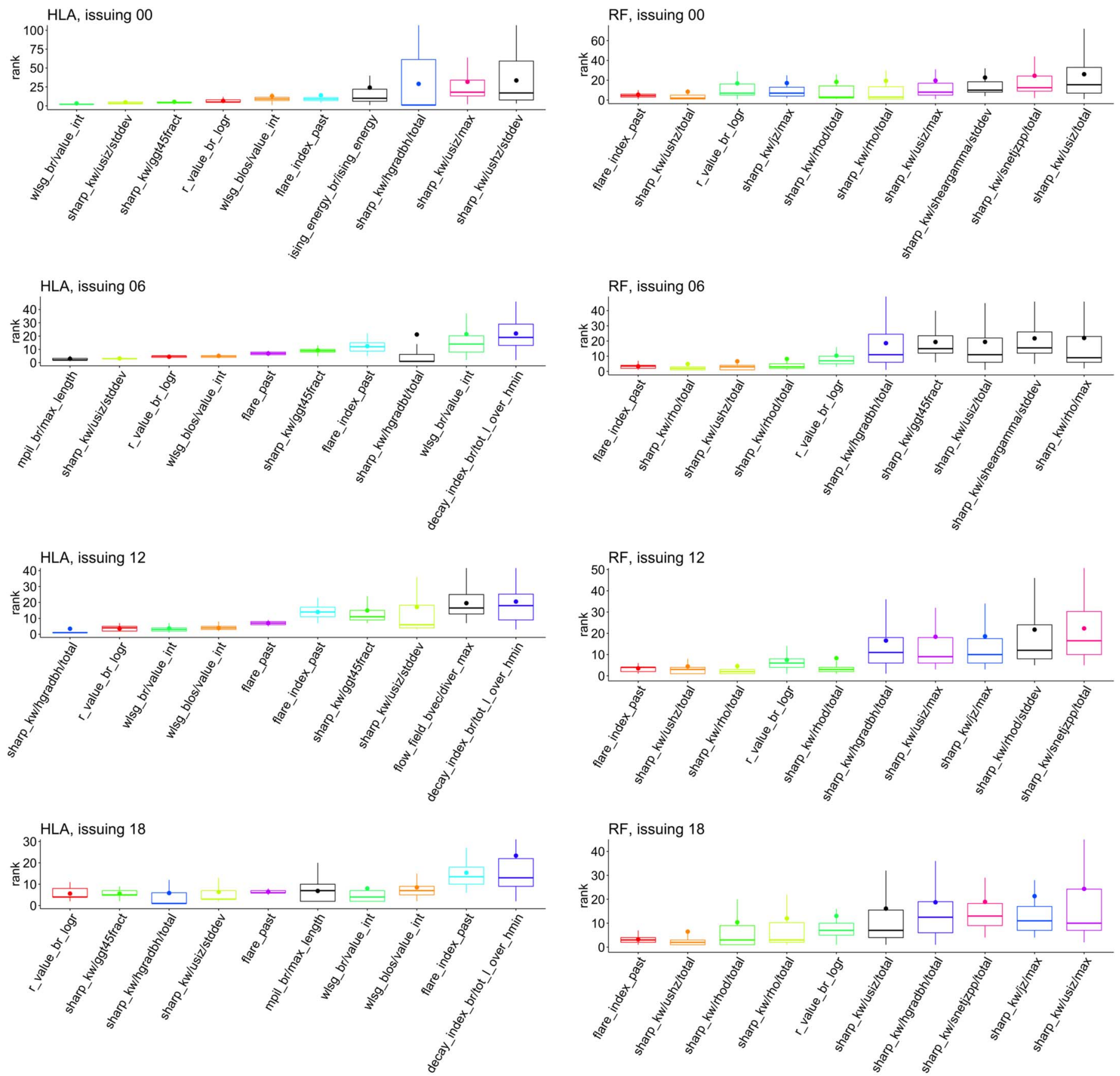

Figure 2. Boxplots of the feature ranks provided by RFE as applied against the outcomes of HLA and RF for the 100 realizations of the training set. The panels show separately the result of the two learning methods (HLA: left column; RF: right column) for the four issuing times considered in the experiment. The focus here is on the prediction of GOES $\mathrm{C} 1+$ flares.

realization, while adding one feature at a time, starting from the feature with the highest ranking, down to the feature with the tenth highest ranking. A given feature has the same color throughout each set of plots, for all issuing times.

In order to have a clearer picture of the features that repeatedly show the highest predictive impact, the histograms in Figure 6 compute the number of times over the four issuing times that each feature appears in the top-10 ranking of training set averages. These plots only present features that reach the top-10 ranking at least twice out of the four issuing times for a given machine-learning method and flare class. For the $\mathrm{C} 1+$ flare prediction (Figure 6; top row) one sees, for example, that the past flare history (flare_index_past) and Schrijver's (Schrijver 2007) $R$-value ( $r$ _value_br_logr) consistently appear for both HLA and RF. This is not the case for the prediction of $\mathrm{M} 1+$ flares (Figure 6; bottom row). It should be noted that the importance of the specific features may only be due to the machine-learning method used; it is their consistency of appearance, however, that is notable.

\section{Discussion of Results}

We first notice that the maximum values of HSS and TSS achieved in Table 1 are distinctly different from one, indicating far from perfect performance. Interestingly, these scores are 

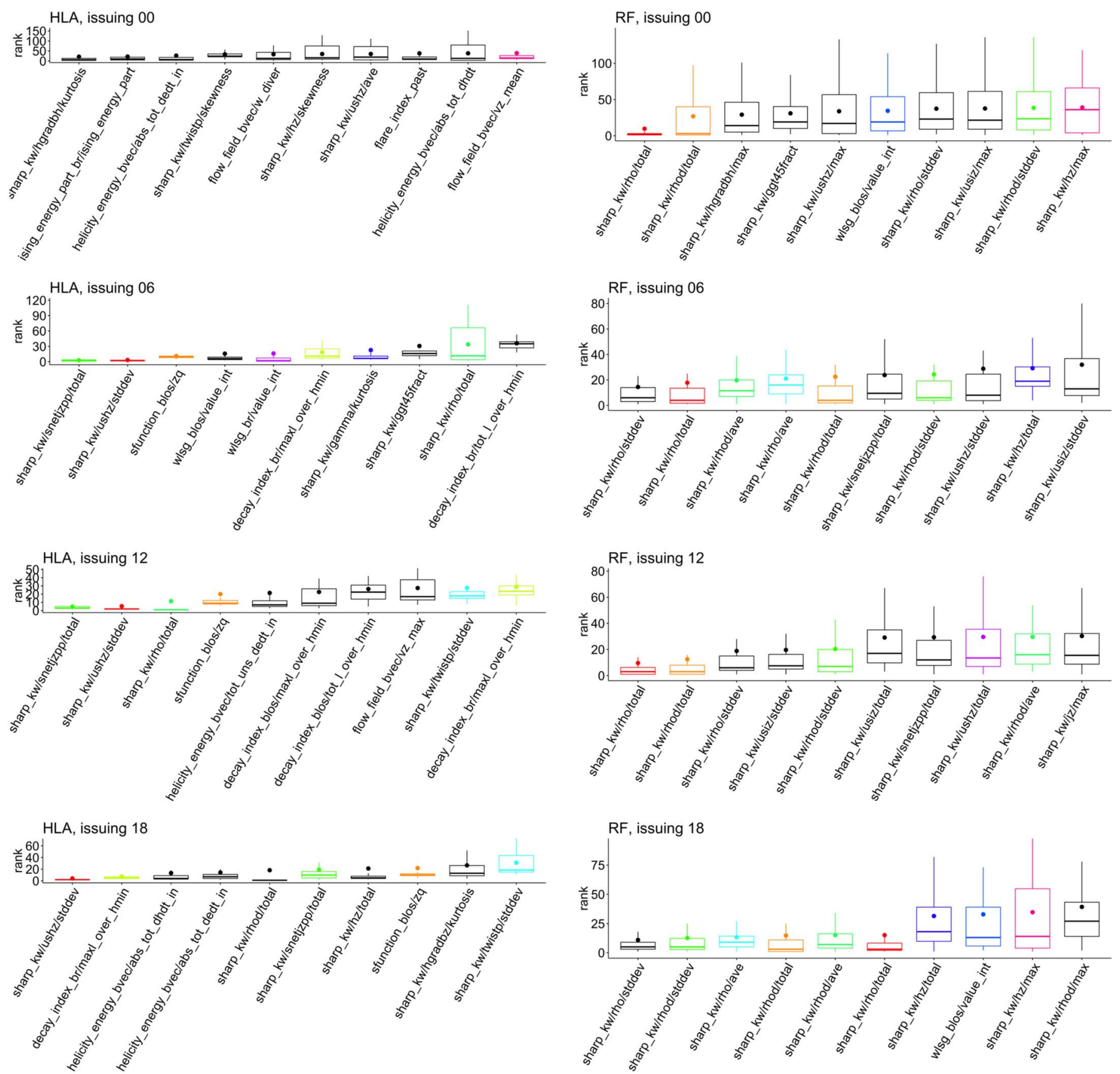

Figure 3. Same as Figure 2, with the focus now being on GOES M1+ flares.

almost systematically smaller than the ones recently achieved by methods illustrated in Bobra \& Couvidat (2015) and Florios et al. (2018) that use input data with a significantly smaller dimensionality. The methods described in those papers are all supervised, utilize features extracted from HMI data, and perform predictions in a $24 \mathrm{~h}$ window. However, the way data preparation is performed and, in particular, how the training set is constructed is significantly different than what is done in the present paper. In particular:

1. The test sets utilized in this work to assess the performances of HLA and RF do not contain feature vectors belonging to ARs with feature vectors contained in the training sets. Instead, the training sets utilized in
Bobra \& Couvidat (2015), and Florios et al. (2018) combined feature vectors belonging to the same ARs in the two sets.

2. We constructed four separate training/testing sets, each corresponding to a specific UT forecast issuing time on all of the days considered. Our results show reasonably consistent forecast performance across these four issuingtime sets. However, the main benefit to this approach is in the interpretation of the feature selection results. Identifying key forecasting features through their appearance in all (or most) of the top-10 feature ranking lists across these four issuing-time sets increases their robustness through temporal consistency. 


\section{issuing 00}

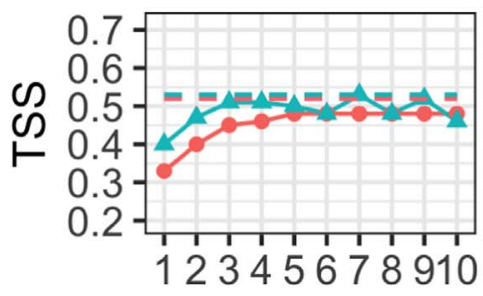

issuing 12

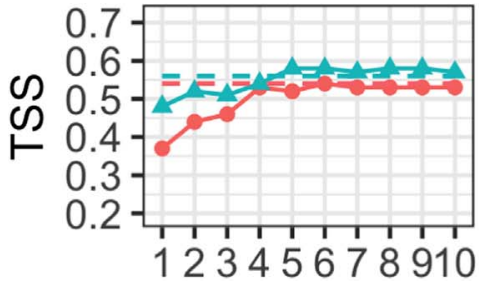

issuing 06

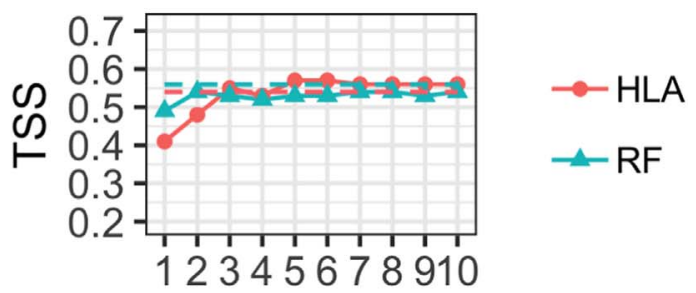

issuing 18

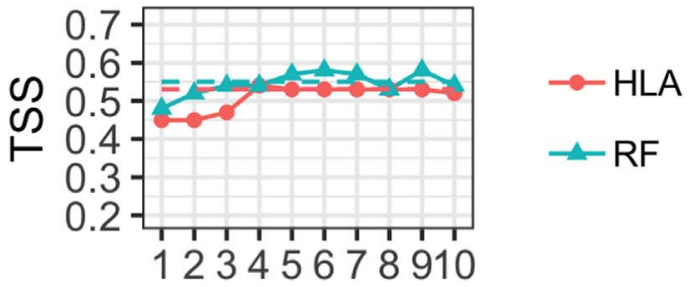

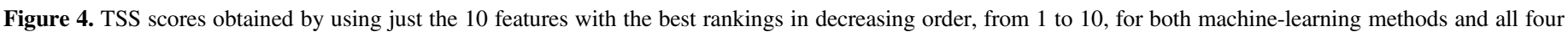

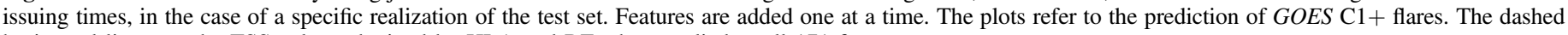
horizontal lines are the TSS values obtained by HLA and RF when applied to all 171 features.

issuing 00

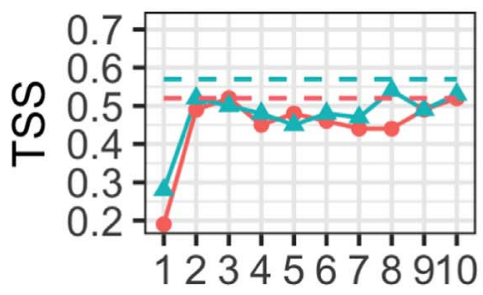

issuing 12

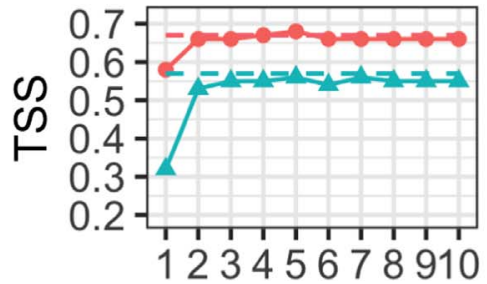

issuing 06

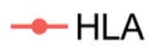

$\triangle \mathrm{RF}$

$\rightarrow$ HLA

$\triangle \mathrm{RF}$

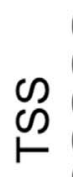

0.7
0.6
0.5
0.4
0.3
0.2

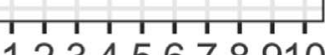

12345678910

issuing 18

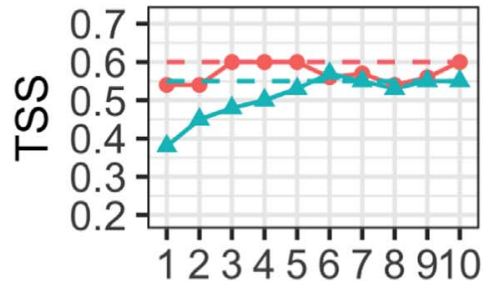

$\rightarrow$ HLA

$\leadsto$ RF

Figure 5. Same as Figure 4, but for GOES M1+ flares.

3. The training set utilized in Florios et al. (2018) is populated with approximately the same number of vectors as the test set, while in our approach (and in the one followed in Bobra \& Couvidat 2015) the machine-learning methods are trained with training sets two times more populated than the test sets, which is more realistic with respect to typical experimental settings.

4. Our prediction methods are optimized using a fuzzy clustering technique, while in the other three cases the input parameters are fixed in such a way to optimize a specific skill score (namely, the TSS).

In order to assess the impact of these differences against the methods' performance, we trained HLA and RF using all 14,931 point-in-time feature vectors distributing them between training and test sets as was done in Bobra \& Couvidat (2015) and Florios et al. (2018). Specifically, we generated the training and test sets focusing on feature vectors instead of on ARs, i.e., we randomly extracted the feature vectors from the database at disposal without imposing any constraint that forbids feature vectors of the same AR to populate both the training and the test set (the two sets are populated as in Bobra \& Couvidat 2015, following a 2:1 proportion). Furthermore, we did not care for time consistency and so we mixed up feature vectors belonging to different issuing times. Finally, the prediction methods are optimized in such a way to maximize the TSS. Table 2 shows that TSS increases significantly in the prediction of $\mathrm{C} 1+$ and $\mathrm{M} 1+$ flares for both 

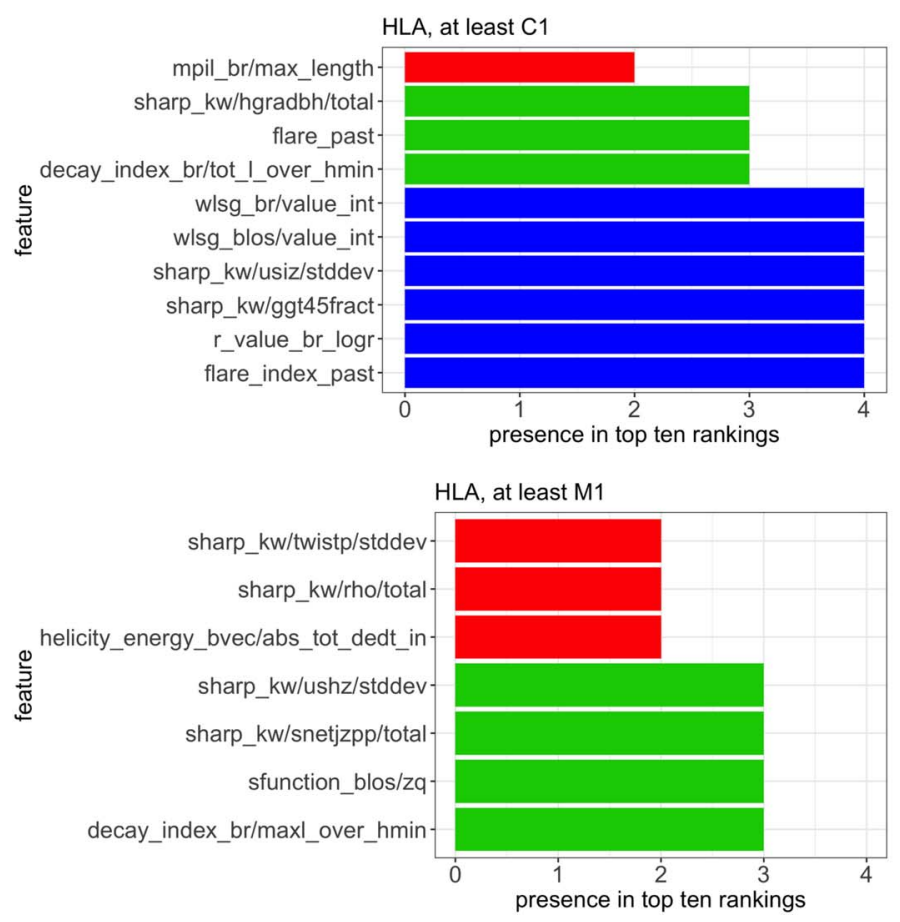
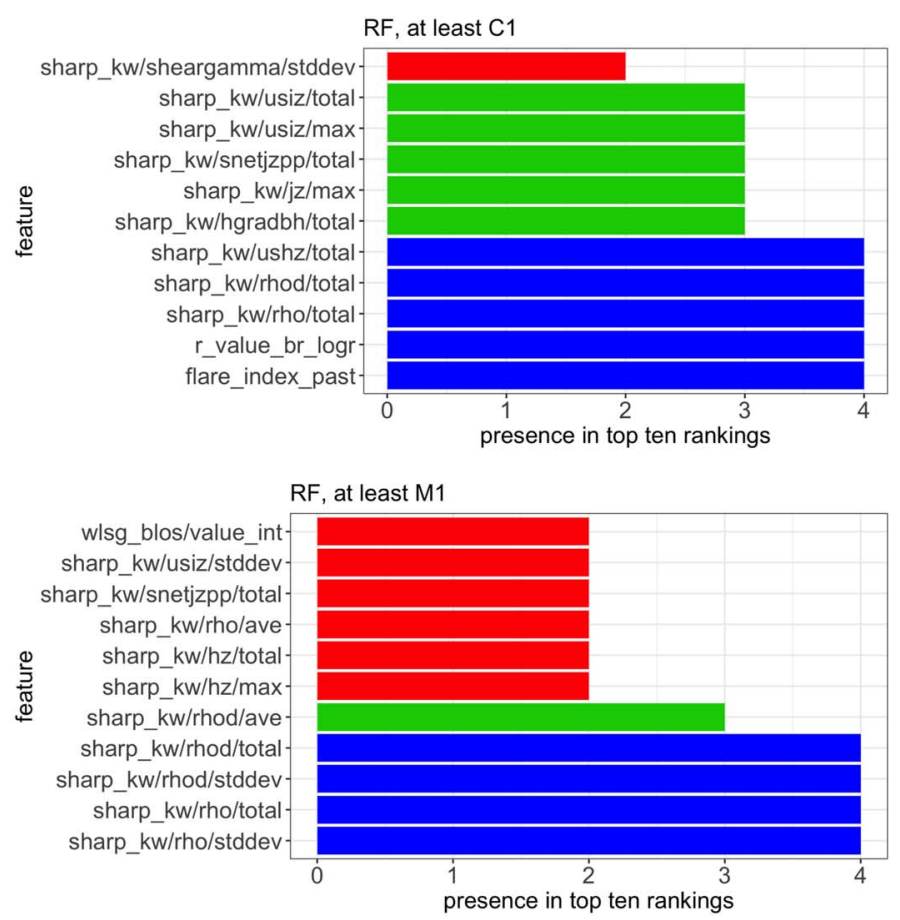

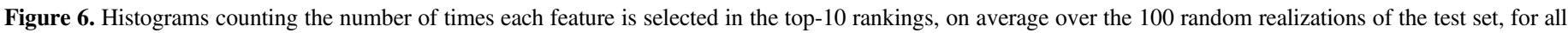

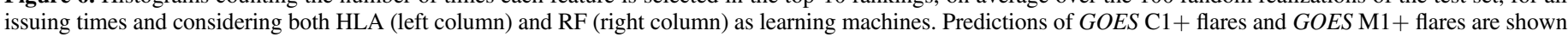
in the top and bottom rows, respectively.

Table 2

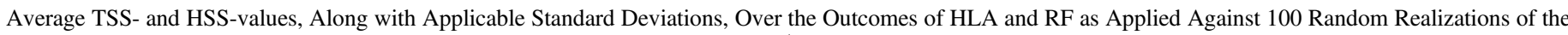
Training/Test Sets

\begin{tabular}{|c|c|c|c|c|}
\hline & Test Set-C1+ & Test Set C1+ & Test Set-M1+ & $\overline{~ T e s t ~ S e t-M 1+}$ \\
\hline & TSS & HSS & TSS & HSS \\
\hline HLA & $0.58 \pm 0.01$ & $0.51 \pm 0.01$ & $0.70 \pm 0.02$ & $0.31 \pm 0.03$ \\
\hline $\mathrm{RF}$ & $0.61 \pm 0.01$ & $0.56 \pm 0.02$ & $0.71 \pm 0.03$ & $0.39 \pm 0.02$ \\
\hline Florios et al. (2018) & $0.60 \pm 0.01$ & $0.59 \pm 0.01$ & $0.74 \pm 0.02$ & $0.49 \pm 0.01$ \\
\hline Bobra \& Couvidat (2015) & $\cdots$ & $\cdots$ & $0.76 \pm 0.04$ & $0.52 \pm 0.04$ \\
\hline
\end{tabular}

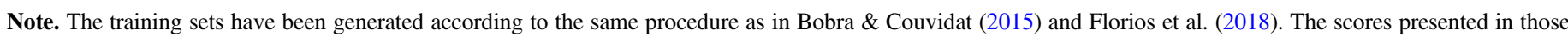
papers are reported in this table.

HLA and RF and also HSS produced by RF becomes larger, although less significantly. These scores are now more in line with the ones obtained in the other two papers, at the same time showing smaller standard deviations. This leads to the conclusion that, not surprisingly, also in flare prediction the biases introduced in the process of training set generation and the way the algorithms are optimized strongly influence the performance of supervised methods.

As far as feature ranking is concerned, it is evident from Figures 2 and 3 that first, features with the best ranking have the smallest standard deviations, so their impact on prediction is consistently high, regardless of the splitting of the data used for training the machine-learning algorithms. In the case of prediction of GOES $\mathrm{C} 1+$ flares, colors largely repeat in all four panels, telling us that features with highest predictive power are common to all issuing times considered. This behavior is not as robust in the case of prediction of GOES M1+ flares, but we are confident that this is a consequence of the lower occurrence rate of $\mathrm{M} 1+$ flares and the resulting variation in the flare /no-flare imbalance ratio of the random training sets. A consistent imbalance ratio is more or less guaranteed for $\mathrm{C} 1+$ flares, whose comprehensive statistics over solar cycle 24 ensure a well-balanced training process.

Figures 4 and 5 show that only a small number of features (up to 10) over the scores of features proposed and/or applied for flare prediction, are sufficient to achieve maximum performance of a given machine-learning method. Notice from these figures that the highest-ranking feature alone (feature 1) suffices to give TSS and HSS values that are at least half of the maxima achieved. Up to the fourth feature, the values of TSS and HSS saturate already, indicating that adding more features will not improve (and may, in fact, be detrimental to) prediction performance. Also, provided that flare statistics are sufficient to deal with the flare/no-flare imbalance ratio in the random selection of training and test sets, these few best-performing features are consistent for a given prediction method. In their study, Bobra \& Couvidat (2015) found that the four most significant features in their analysis were the total unsigned current helicity, total magnitude of the Lorentz force, total photospheric magnetic free energy density, and unsigned 
vertical current. In our study, Figure 6 shows that features associated with the unsigned vertical current (i.e., total, maximum, standard deviation) are among the most temporally consistent of our best-performing features, particularly for $\mathrm{C} 1+$ flares (its standard deviation is in the top-ten features of all four UT issuing times for Hybrid LASSO, while its total and maximum are in the top-10 of three of the four UT issuing times for RF) and less so for M1+ flares (its standard deviation is in the top-10 for two of the four UT issuing times). However, the same figure shows that these predictors may well change from method to method, which hampers efforts to understand physically why some features work better than others. Best performers appear to change also for the prediction of different flare classes, which is a very interesting finding that undoubtedly warrants additional investigation in the future.

\section{Conclusions}

This study employs the highest-dimension data set of prediction features to date in regards to solar flare forecasting, while it shows TSS values similar to the better performing region-by-region forecasting systems in the literature. This point taken, the actual HSS and TSS values are not identical to - and may even be somewhat lower than-the respective values reported in Bobra \& Couvidat (2015) and in Florios et al. (2018), with the latter study using RF applied to FLARECAST data, as well. The reason is that training and testing of machine-learning methods in the present study were not only performed on nonoverlapping data as in previous studies, but even the solar ARs selected for training and testing were different. This conclusion seems in line with the considerations contained in Camporeale (2019), whose careful assessment of the causality issue identifies this as one of the crucial aspects impacting the forecasting performances.

The rationale for using hybrid LASSO and RF in this work is these methods' ability to perform feature ranking via RFE, among other methods (i.e., Fisher's score, Gini index, etc.). However, there are 26 machine-learning methods implemented in FLARECAST. Their definitive evaluation is in progress, so the values of pertinent skill scores may well increase in future studies utilizing FLARECAST data, in the search for finding the optimal machine-learning method(s) for the near-realtime FLARECAST forecasting service. We also understand that a meaningful methodological next step would be to introduce deep learning methods in the pipeline. However, interestingly, the use of these more modern approaches in flare forecasting does not necessarily imply significantly higher skill scores (see, e.g., (Nishizuka et al. 2018), where TSS and HSS for the prediction of $\mathrm{M} 1+$ flares are reported as 0.80 and 0.26 , respectively).

What will, most likely, not change in the foreseeable future are the following two core conclusions of this work.
First, the current range of properties that have been extracted from the HMI magnetograms show significant redundancy and no more than 10 features contained in these properties are sufficient to allow machine-learning methods to achieve maximum performance.

Second, and perhaps foremost, the maximum values of HSS and TSS achieved are distinctly different from one, indicating far from perfect performance. In physical terms, even using the largest flare prediction data volume assembled to date, we have not managed to substantially surpass the performance of a random-chance forecast (as shown by HSS) or to substantially increase the probability of detection $(0.57-0.65$ for $\mathrm{C} 1+$ ) despite an encouragingly low probability of false detection $(\sim 0.10)$. The latter two compete against each other to result in TSS. This is equivalent to saying that flare prediction remains probabilistic, rather than binary yes/no with a perfect performance. The core reasons for this may be multiple: first, we only rely on photospheric magnetic field data, but flares occur above the line-tied photosphere in the low solar corona. Second, flares may well be intrinsically stochastic phenomena, as adopted in a long-standing working hypothesis (Rosner \& Vaiana 1978), shown conclusively by the flares' timedependent Poisson waiting times (Crosby et al. 1998; Wheatland \& Litvinenko 2002) and interpreted physically via the concept of self-organized criticality (Lu \& Hamilton 1991; Lu et al. 1993; Vlahos et al. 1995)—see also Aschwanden et al. (2016) for a comprehensive review.

This study was enabled by the European Union's Horizon 2020 Research and Innovation Action Flare Likelihood And Region Eruption foreCASTing (FLARECAST) project, under grant agreement No. 640216. All authors warmly acknowledge the support of the FLARECAST project.

\section{Appendix}

Here we provide details of the FLARECAST feature labels used in this work, with short descriptions and references to their original definition/implementation (or, e.g., detection methods used in their calculation). Features are grouped in the following manner: Table 3 contains those features derived from $B_{\text {los }}$ only and $B_{\text {radial }}$ only (Georgoulis 2005, 2012; Georgoulis \& Rust 2007; Schrijver 2007; Falconer et al. 2008; Hewett et al. 2008; Ahmed et al. 2010; Mason \& Hoeksema 2010; Georgoulis et al. 2012; Zuccarello et al. 2014; Guerra et al. 2015; Kontogiannis et al. 2018); Table 4 contains those features requiring all three vectormagnetic field components (Kusano et al. 2002; Schuck 2008); Table 5 contains only those features related to the total and mean quantities provided as the SHARP keywords of Bobra et al. (2014). 
Table 3

FLARECAST $B \_l o s-$ and $B \_r$-derived Feature List with Short Descriptions

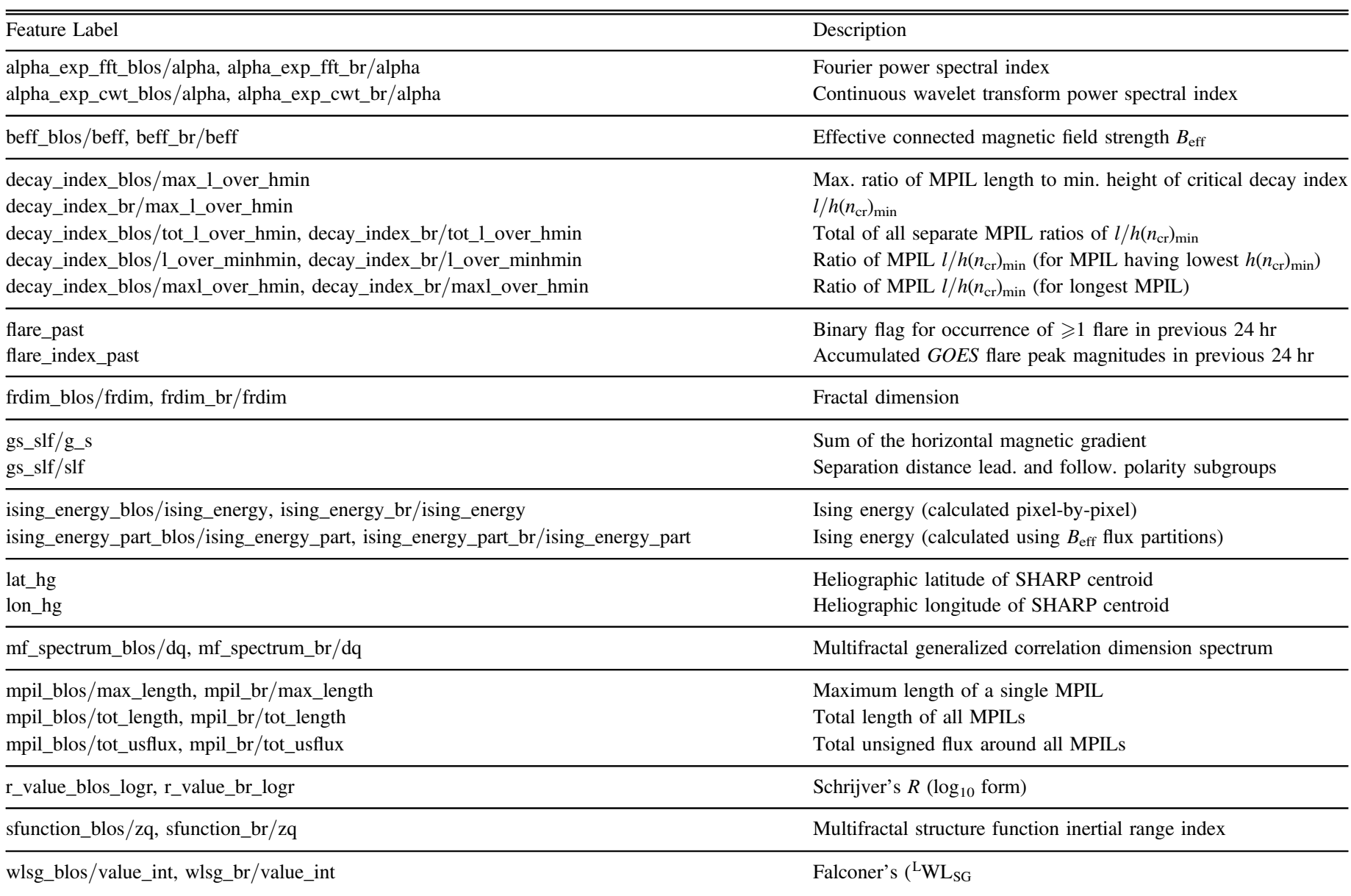

Table 4

FLARECAST $B_{r, \theta, \phi}$-derived Feature List with Short Descriptions

\begin{tabular}{ll}
\hline \hline Feature Label & Description \\
\hline flow_field_bvec/diver, flow_field_bvec/diver_max,flow_field_bvec/diver_mean & Flow field divergence (total, maximum, mean) \\
\hline flow_field_bvec/shear, flow_field_bvec/shear_max, flow_field_bvec/shear_mean & Flow field shear (total, maximum, mean) \\
\hline flow_field_bvec/v_mean, flow_field_bvec/v_median & Flow field total velocity magnitude (mean, median) \\
\hline flow_field_bvec/vz_max,flow_field_bvec/vz_mean & Flow field vertical velocity magnitude (mean, median) \\
\hline flow_field_bvec/w_diver,flow_field_bvec/w_diver_max, flow_field_bvec/w_diver_mean & Flux-weighted flow field divergence (total, maximum, mean) \\
\hline flow_field_bvec/w_shear,flow_field_bvec/w_shear_max, flow_field_bvec/w_shear_mean & Flux-weighted flow field shear (total, maximum, mean) \\
\hline helicity_energy_bvec/abs_tot_dedt & Abs. val. net vertical Poynting flux \\
helicity_energy_bvec/abs_tot_dedt_in & Abs. val. net vertical Poynting flux (emerg. comp.) \\
helicity_energy_bvec/abs_tot_dedt_sh & Abs. val. net vertical Poynting flux (shear. comp.) \\
helicity_energy_bvec/abs_tot_dedt_in_plus_sh & Emerg. + shear. abs. values net vertical Poynting flux \\
\hline helicity_energy_bvec/abs_tot_dhdt & Abs. val. net vertical helicity flux \\
helicity_energy_bvec/abs_tot_dhdt_in & Abs. val. net vertical helicity flux (emerg. comp.) \\
helicity_energy_bvec/abs_tot_dhdt_sh & Abs. val. net vertical helicity flux (emerg. comp.) \\
helicity_energy_bvec/abs_tot_dhdt_in_plus_sh & Emerg. + shear. abs. values net vertical helicity flux \\
\hline helicity_energy_bvec/tot_uns_dedt & Total unsigned vertical Poynting flux \\
helicity_energy_bvec/tot_uns_dedt_in & Tot. unsign. vertical Poynting flux (emerg. comp.) \\
helicity_energy_bvec/tot_uns_dedt_sh & Tot. unsign. vertical Poynting flux (shear. comp.) \\
\hline
\end{tabular}


Table 4

(Continued)

Feature Label

helicity_energy_bvec/tot_uns_dhdt

helicity_energy_bvec/tot_uns_dhdt_in

helicity_energy_bvec/tot_uns_dhdt_sh

nn_currents/tot_us_cur
Description

Tot. unsign. vertical helicity flux

Tot. unsign. vertical helicity flux (emerg. comp.)

Tot. unsign. vertical helicity flux (shear. comp.)

Total unsigned nonneutralized currents

Table 5

FLARECAST SHARP-keyword Related Feature List with Short Descriptions

\begin{tabular}{ll}
\hline \hline Feature Label & Description \\
\hline sharp_kw/gamma/ave, sharp_kw/gamma/stddev & Field inclin. ang. (mean, st. dev.) \\
sharp_kw/gamma/skewness, sharp_kw/gamma/kurtosis & Field inclin. ang. (skewn., kurt.) \\
sharp_kw/gamma/total, sharp_kw/gamma/max, sharp_kw/gamma/median & Field inclin. ang. (tot., max., med.) \\
\hline
\end{tabular}

sharp_kw/ggt45fract

$\%$ tot. area with shear angle $>45^{\circ}$

sharp_kw/hgradbh/ave, sharp_kw/hgradbh/stddev

sharp_kw/hgradbh/skewness, sharp_kw/hgradbh/kurtosis

sharp_kw/hgradbh/total, sharp_kw/hgradbh/max, sharp_kw/hgradbh/median

Horiz. grad. $B_{\text {hor }}$ (mean, st. dev.)

Horiz. grad. $B_{\text {hor }}$ (skewn., kurt.)

Horiz. grad. $B_{\text {hor }}$ (tot, max, med)

sharp_kw/hgradbt/ave, sharp_kw/hgradbt/stddev

sharp_kw/hgradbt/skewness, sharp_kw/hgradbt/kurtosis

Horiz. grad. $B_{\text {tot }}$ (mean, st. dev.)

sharp_kw/hgradbt/total, sharp_kw/hgradbt/max, sharp_kw/hgradbt/median

Horiz. grad. $B_{\text {tot }}$ (skewn., kurt.)

Horiz. grad. $B_{\text {tot }}$ (tot., max., med.)

sharp_kw/hgradbz/ave, sharp_kw/hgradbz/stddev

sharp_kw/hgradbz/skewness, sharp_kw/hgradbz/kurtosis

sharp_kw/hgradbz/total, sharp_kw/hgradbz/max, sharp_kw/hgradbz/median

Horiz. grad. $B_{r}$ (mean, st. dev.)

Horiz. grad. $B_{r}$ (skewn., kurt.)

Horiz. grad. $B_{r}$ (tot., max., med.)

sharp_kw/hz/ave, sharp_kw/hz/stddev

sharp_kw/hz/skewness, sharp_kw/hz/kurtosis

sharp_kw/hz/total, sharp_kw/hz/max, sharp_kw/hz/median

Vert. curr. hel. (mean, st. dev.)

Vert. curr. hel. (skewn., kurt.)

Vert. curr. hel. (tot., max., med.)

sharp_kw/jz/ave, sharp_kw/jz/stddev

sharp_kw/jz/skewness, sharp_kw/jz/kurtosis

sharp_kw/jz/total, sharp_kw/jz/max, sharp_kw/jz/median

Vert. curr. (mean, st. dev.)

Vert. curr. (skewn., kurt.)

Vert. curr. (tot., max., med.)

sharp_kw/rho/ave, sharp_kw/rho/stddev

sharp_kw/rho/skewness, sharp_kw/rho/kurtosis

sharp_kw/rho/total, sharp_kw/rho/max, sharp_kw/rho/median

Photosph. excess magn. en. (mean, st. dev.)

Photosph. excess magn. en. (skewn., kurt.)

Photosph. excess magn. en. (tot., max., med.)

sharp_kw/rhod/ave, sharp_kw/rhod/stddev

sharp_kw/rhod/skewness, sharp_kw/rhod/kurtosis

sharp_kw/rhod/total, sharp_kw/rhod/max, sharp_kw/rhod/median

Photosph. excess magn. en. dens. (mean, st. dev.)

Photosph. excess magn. en. dens. (skewn., kurt.)

Photosph. excess magn. en. dens (tot., max., med.)

sharp_kw/sflux/ave, sharp_kw/sflux/stddev

sharp_kw/sflux/skewness, sharp_kw/sflux/kurtosis

sharp_kw/sflux/total, sharp_kw/sflux/max, sharp_kw/sflux/median

Signed flux (mean, st. dev.)

Signed flux (skewn., kurt.)

Signed flux (tot., max., med.)

sharp_kw/sheargamma/ave, sharp_kw/sheargamma/stddev

sharp_kw/sheargamma/skewness, sharp_kw/sheargamma/kurtosis

sharp_kw/sheargamma/total, sharp_kw/sheargamma/max, sharp_kw/sheargamma/median

$B_{\text {tot }}$ shear angle (mean, st. dev.)

$B_{\text {tot }}$ shear angle (skewn., kurt.)

$B_{\text {tot }}$ shear angle (tot., max., med.)

sharp_kw/snetjzpp/total

Sum abs. val. net currents per polarity

sharp_kw/twistp/ave, sharp_kw/twistp/stddev, sharp_kw/twistp/skewness, sharp_kw/twistp/kurtosis

sharp_kw/twistp/total, sharp_kw/twistp/max, sharp_kw/twistp/median

Twist parameter (mean, st. dev., skewn., kurt.)

Twist parameter (tot., max., med.)

sharp_kw/usflux/ave, sharp_kw/usflux/stddev, sharp_kw/usflux/skewness, sharp_kw/usflux/kurtosis

Uns. flux (mean, st. dev., skewn., kurt.)

sharp_kw/usflux/total, sharp_kw/usflux/max, sharp_kw/usflux/median

Uns. flux (tot., max., med.)

sharp_kw/ushz/ave, sharp_kw/ushz/stddev, sharp_kw/ushz/skewness, sharp_kw/ushz/kurtosis sharp_kw/ushz/total, sharp_kw/ushz/max, sharp_kw/ushz/median

Uns. vert. curr. hel. (mean, st. dev., skewn., kurt.)

Uns. vert. curr. hel. (tot., max., med.)

sharp_kw/usiz/ave, sharp_kw/usiz/stddev, sharp_kw/usiz/skewness, sharp_kw/usiz/kurtosis

sharp_kw/usiz/total, sharp_kw/usiz/max, sharp_kw/usiz/median

Uns. vert. curr. (mean, st. dev., skewn., kurt.)

Uns. vert. curr. (tot., max., med.) 


\section{ORCID iDs}

Federico Benvenuto (i) https://orcid.org/0000-0002-4776-0256

D. Shaun Bloomfield (i) https://orcid.org/0000-0002-4183-9895

Manolis K. Georgoulis (i https://orcid.org/0000-0001-6913-1330

\section{References}

Ahmed, O. W., Qahwaji, R., Colak, T., et al. 2013, SoPh, 283, 157

Ahmed, O. W., Qahwaji, R., Colak, T., Dudok De Wit, T., \& Ipson, S. 2010 , Vis. Comp., 26, 385

Aschwanden, M. J., Crosby, N. B., Dimitropoulou, M., et al. 2016, SSRv, 198,47

Barnes, G., \& Leka, K. D. 2008, ApJL, 688, L107

Barnes, G., Leka, K. D., Schrijver, C. J., et al. 2016, ApJ, 829, 89

Benvenuto, F., Piana, M., Campi, C., \& Massone, A. M. 2018, ApJ, 853, 90

Benz, A. O. 2017, LRSP, 14, 2

Bezdek, J. C., Ehrlich, R., \& Full, W. 1984, CG, 10, 191

Bloomfield, D. S., Higgins, P. A., McAteer, R. T. J., \& Gallagher, P. T. 2012, ApJL, 747, L41

Bobra, M. G., \& Couvidat, S. 2015, ApJ, 798, 135

Bobra, M. G., Sun, X., Hoeksema, J. T., et al. 2014, SoPh, 289, 3549

Breiman, L. 2001, Machine Learning, 45, 5

Camporeale, E. 2019, arXiv:1903.05192

Colak, T., \& Qahwaji, R. 2009, SpWea, 7, S06001

Cortes, C., \& Vapnik, V. 1995, Machine Learning, 20, 273

Crosby, N., Vilmer, N., Lund, N., \& Sunyaev, R. 1998, A\&A, 334, 299

Evgeniou, T., Pontil, M., \& Poggio, T. 2000, Adv. Comput. Math., 13, 1

Falconer, D. A., Moore, R. L., \& Gary, G. A. 2008, ApJ, 689, 1433

Florios, K., Kontogiannis, I., Park, S.-H., et al. 2018, SoPh, 293, 28

Gallagher, P. T., Moon, Y. J., \& Wang, H. 2002, SoPh, 209, 171

Georgoulis, M. K. 2005, SoPh, 228, 5

Georgoulis, M. K. 2012, SoPh, 276, 161

Georgoulis, M. K., \& Rust, D. M. 2007, ApJL, 661, L109

Georgoulis, M. K., Titov, V. S., \& Mikić, Z. 2012, ApJ, 761, 61

Guerra, J. A., Park, S. H., Gallagher, P. T., et al. 2018, SoPh, 293, 9

Guerra, J. A., Pulkkinen, A., Uritsky, V. M., \& Yashiro, S. 2015, SoPh, 290, 335

Guyon, I., Weston, J., Barnhill, S., \& Vapnik, V. 2002, Machine Learning, 46, 389

Hewett, R. J., Gallagher, P. T., McAteer, R. T. J., et al. 2008, SoPh, 248, 311
Higgins, P. A., Gallagher, P. T., McAteer, R. T. J., \& Bloomfield, D. S. 2011, AdSpR, 47, 2105

Huang, X., Wang, H., Xu, L., et al. 2018, ApJ, 856, 7

Kontogiannis, I., Georgoulis, M. K., Park, S.-H., \& Guerra, J. A. 2018, SoPh, 293, 96

Korsos, M. B., \& Erdelyi, R. 2016, ApJ, 823, 153

Kusano, K., Maeshiro, T., Yokoyama, T., \& Sakurai, T. 2002, ApJ, 577, 501

Leka, K. D., Park, S.-H., Kusano, K., et al. 2019a, ApJS, 243, 36

Leka, K. D., Park, S.-H., Kusano, K., et al. 2019b, ApJ, 881, 101

Li, R., Cui, Y., He, H., \& Wang, H. 2008, AdSpR, 42, 1469

Li, R., Wang, H. N., He, H., Cui, Y. M., \& Du, Z. L. 2007, ChJAA, 7, 441

Lu, E. T., \& Hamilton, R. J. 1991, ApJL, 380, L89

Lu, E. T., Hamilton, R. J., McTiernan, J. M., \& Bromund, K. R. 1993, ApJ, 412, 841

Mason, J. P., \& Hoeksema, J. T. 2010, ApJ, 723, 634

Massone, A. M., Piana, M. \& The FLARECAST Team 2018, in Machine Learning for Flare Forecasting, Machine Learning Techniques for Space Weather, ed. E. Camporeale, S. Wing, \& J. R. Johnson (Amsterdam: Elsevier)

McCloskey, A. E., Gallagher, P. T., \& Bloomfield, D. S. 2017, JSWSC, 8, A34

Murray, S. A., Bingham, S., Sharpe, M., \& Jackson, D. R. 2017, SpWea, 15,577

Nishizuka, N., Sugiura, K., Kubo, Y., Den, M., \& Ishii, M. 2018, ApJ, 858,113

Park, E., Moon, Y. J., Shin, S., et al. 2018, ApJ, 869, 91

Priest, E. R., \& Forbes, T. G. 2002, A\&ARv, 10, 313

Rosner, R., \& Vaiana, G. S. 1978, ApJ, 222, 1104

Sadykov, V. M., \& Kosovichev, A. G. 2017, ApJ, 849, 148

Scherrer, P. H., Schou, J., Bush, R. I., et al. 2012, SoPh, 275, 207

Schrijver, C. J. 2007, ApJL, 655, L117

Schuck, P. W. 2008, ApJ, 683, 1134

Schwenn, R. 2006, LRSP, 3, 2

Tibshirani, R. 1996, J. Royal. Stat. Soc. B, 58, 267

Vlahos, L., Georgoulis, M., Kluiving, R., \& Paschos, P. 1995, A\&A, 299, 897

Wang, H. N., Cui, Y. M., Li, R., Zhang, L. Y., \& Han, H. 2008, AdSpR, 42,1464

Wheatland, M. S., \& Litvinenko, Y. E. 2002, SoPh, 211, 255

Wu, T. T., Chen, Y. F., Hastie, T., Sobel, E., \& Lange, K. 2009, Bioinformatics, 25, 714

Youden, W. J. 1950, Cancer, 3, 32

Yu, D., Huang, X., Wang, H., \& Cui, Y. 2009, SoPh, 255, 91

Zuccarello, F. P., Seaton, D. B., Mierla, M., et al. 2014, ApJ, 785, 88 